\title{
Mathematical phantoms development for computer simulation of the patient examination procedure by a positron emission tomography method
}

\author{
M. Ondar ${ }^{1,2 *}$, N. Denisova ${ }^{1}$ \\ ${ }^{1}$ Institute of Theoretical and Applied Mechanics SB RAS, Novosibirsk, Russia \\ ${ }^{2}$ Novosibirsk State Technical University, Novosibirsk, Russia \\ *e-mail: ondar_marina93@mail.ru
}

Key words: positron emission tomography (PET), radiopharmaceutical, anthropomorphic phantoms

Motivation and Aim: The method of positron emission tomography (PET) is the "gold standard" in oncology. A $90 \%$ of PET method studies use a radiopharmaceutical fluorodeoxyglucose (FDG) with a property of accumulating in malignant tumors. Therefore, the PET method makes it possible to distinguish the benign and malignant formations. A three-dimensional image creation is the result of solving the ill-posed problem of reconstruction. It is well known, that in solving such problems there are artifacts related to instability of the solution and they are dependent on the source function. The most effective way is the method of mathematical modeling to investigate the causes of the artifacts appearance. The modeling procedure includes the next steps: 1) model creation - source function, 2) raw data calculation using created model, 3) image acquisition based on the chosen method for solving the ill-posed reconstruction problem, 4) comparison of the obtained image with a specified source function and errors analysis of reconstruction. Because of radiation exposure, it is impossible to conduct research in humans in the field of nuclear medicine. To simulate the PET procedure, it is necessary to develop anthropomorphic phantoms simulating the radiopharmaceutical distribution in the patient's organs. The aim of this work is the development of anthropomorphic phantoms describing the distribution of the FDG radiopharmaceutical for mathematical modeling of the procedure for acquisition the "whole body image" using a PET method. Methods and Algorithms: The anthropomorphic phantoms development is carried out on the basis equations of analytic geometry that describe spatial geometric figures using Boolean logical operators.

Results: Amathematical model describing the distribution of the FDG radiopharmaceutical throughout the body is developed to simulate the procedure for examining cancer patients using the PET method to diagnose the presence or absence of metastases.

Conclusion: These studies are part of the work of the scientific group of ITAM SB RAS to improve the methods of reconstruction of PET images. These phantoms will be used for the subsequent computer simulation of PET: the raw data calculation recorded by a gamma camera; images reconstruction using standard algorithms.

Acknowledgements: The work was supported by grant from RFBR (No. 17-52-14004). 